\title{
METRIC THEORY OF SEMIALGEBRAIC CURVES
}

\author{
Lev BIRBRAIR* \\ and Alexandre C.G. FERNANDES
}

\begin{abstract}
We present a complete bi-Lipschitz classification of germs of semialgebraic curves (semialgebraic sets of the dimension one). For this purpose we introduce the so-called Hölder Semicomplex, a biLipschitz invariant. Hölder Semicomplex is the collection of all first exponents of Newton-Puiseux expansions, for all pairs of branches of a curve. We prove that two germs of curves are bi-Lipschitz equivalent if and only if the corresponding Hölder Semicomplexes are isomorphic. We also prove that any Hölder Semicomplex can be realized as a germ of some plane semialgebraic curve. Finally, we compare these Hölder Semicomplexes with Hölder Complexescomplete bi-Lipschitz invariant of two-dimensional semialgebraic sets.
\end{abstract}

\section{Introduction}

In this paper, we are going to study singular semialgebraic curves (onedimensional semialgebraic sets) from the metric viewpoint. Some recent papers on the metric theory of semialgebraic sets were devoted to intrinsic metric properties of semialgebraic surfaces (see [2],[1],[6]). One of the main questions of this theory is the question of bi-Lipschitz classification. This question was investigated in [1], for germs of semialgebraic surfaces, with respect to the intrinsic (length) metric. The problem of bi-Lipschitz classification with respect to the induced (euclidian) metric is more complicated. Namely, if two semialgebraic sets are bi-Lipschitz

•Partially supported by CMP by grant N300985/93-2

1991 Mathematics Subject Classification: 14P25.

Servicio de Publicaciones. Universidad Complutense. Madrid, 2000 
equivalent with respect to the euclidian metric they are bi-Lipschitz equivalent with respect to the length metric, but the converse is not true.

The main result of the paper is a bi-Lipschitz classification of germs of semialgebraic curves with respect to the euclidian metric. We construct an invariant - the so-called Hölder Semicomplex. This invariant is obtained in the following way. Take two branches $X_{1}$ and $X_{2}$ of a semialgebraic curve $X$ at the point $x_{0} \in X$. Let $x_{1}(r) \in X_{1}$ and $x_{2}(r) \in X_{2}$ be two points on these branches such that $d_{\text {ind }}\left(x_{1}(r), x_{0}\right)=$ $d_{\text {ind }}\left(x_{2}(r), x_{0}\right)=r$. The function $d_{\text {ind }}\left(x_{1}(r), x_{2}(r)\right)$ (the induced metric) is semialgebraic and, thus, admits a Newton-Puiseux expansion at 0 . Hölder Semicomplex is the collection of all first exponents of NewtonPuiseux expansions, for all pairs of branches. Note, that this procedure is closely related to the calculation of Lojasiewicz exponent [4].

We prove that Hölder Semicomplex is a complete invariant. It means that germs of two semialgebraic curves are bi-Lipschitz equivalent if their Hölder Semicomplexes are combinatorially equivalent (isomorphic). We prove (Theorem 4.1), moreover, that, for every Hölder Semicomplex, there exists a germ of a plane semialgebraic curve realizing it (i.e. the Hölder Semicomplex of this curve coincides with the given one) (Theorem 5.1).

We study in section 6 some relations between Hölder Complexes [1] and Hölder Semicomplexes. Hölder Complexes defined in [1] are complete invariants of germs of semialgebraic surfaces. We prove that if a two-dimensional set $X$ is normally embedded at $x_{0}$ (i.e. intrinsic and induced metrics are bi-Lipschitz equivalent [3]) then the Hölder Semicomplex of the germ of $\operatorname{Sing}(X)$ at $x_{0}$ is completely determined by the Hölder Complex of $X$ at $x_{0}$ (Theorem 6.2). Some examples presented in the section 6 show that this result does not remain true if $X$ is not normally embedded.

Note that all results of the paper are hold for semianalytic set. As a consequence of this observation we obtain that every germ of a semianalytic curve is bi-Lipschitz equivalent to a germ of some semialgebraic curve (Corollary 5.2).

The authors are grateful to Maria Aparecida Ruas, Jean-Jacques Risler and the anonymous referee for very useful comments. 


\section{Hölder Semicomplexes}

Definition 1. A complete finite graph $\Gamma$ with a rational valued function $\alpha: E_{\Gamma} \rightarrow \mathbb{Q} \cap[1, \infty)$ defined on the set of edges $E_{\Gamma}$ of $\Gamma$ is called a Hölder Semicomplex if $\alpha$ satifies the following:

Isosceles Property. For every three vertices $a_{1}, a_{2}, a_{3} \in \Gamma$, we have: if $\alpha\left(a_{1}, a_{2}\right) \leq \alpha\left(a_{2}, a_{3}\right) \leq \alpha\left(a_{1}, a_{3}\right)$ then $\alpha\left(a_{1}, a_{2}\right)=\alpha\left(a_{2}, a_{3}\right)$. (Note that, since $\Gamma$ is a complete graph, any edge is completely determined by two vertices).

Remark 1. A Hölder Semicomplex can be defined in the following equivalent way. Let $A$ be a finite set with a symmetric function $\alpha$ : $A \times A-\operatorname{diag}(A \times A) \rightarrow \mathbb{Q} \cap[1, \infty)$. If $\alpha$ satisfies the isosceles property then the pair $(A, \alpha)$ can be identified with a Hölder Semicomplex.

Remark 2. Consider a function $d: A \times A \rightarrow \mathbb{Q}$ such that

$$
d\left(a_{1}, a_{2}\right)=0 \quad \text { if } \quad a_{1}=a_{2} \quad \text { and } \quad d\left(a_{1}, a_{2}\right)=\frac{1}{\alpha\left(a_{1}, a_{2}\right)} \quad \text { otherwise. }
$$

Then $(A, d)$ is an ultrametric space if and only if $(A, \alpha)$ is a Hölder Semicomplex.

Definition 2. Two Hölder Semicomplexes $\left(\Gamma_{1}, \alpha_{1}\right)$ and $\left(\Gamma_{2}, \alpha_{2}\right)$ are called isomorphic (or combinatorially equivalent) if there exists a graph isomorphism $h: \Gamma_{1} \rightarrow \Gamma_{2}$ such that, for each pair of vertices $a_{1}, a_{2}$ of $\Gamma_{1}$, we have:

$$
\alpha_{2}\left(h\left(a_{1}\right), h\left(a_{2}\right)\right)=\alpha_{1}\left(a_{1}, a_{2}\right) .
$$

Definition 3. A morphism of $\left(A_{1}, \alpha_{1}\right)$ to $\left(A_{2}, \alpha_{2}\right)$ is a map $\mathfrak{m}: A_{1} \rightarrow A_{2}$ such that, for all $a_{1}, a_{2} \in A_{1}$,

$$
\alpha_{2}\left(\mathfrak{m}\left(a_{1}\right), \mathfrak{m}\left(a_{2}\right)\right) \geq \alpha_{1}\left(a_{1}, a_{2}\right) \quad \text { if } \quad \mathfrak{m}\left(a_{1}\right) \neq \mathfrak{m}\left(a_{2}\right) .
$$

Thus, we obtain that the set of all Hölder Semicomplexes is a category, the isomorphism defined in Definition 2 is an isomorphism in this category and Remark 2 defines a functor from this category to the category of metric spaces. 


\section{Semialgebraic curves and Hölder Semicom- plex}

Let $X \subset \mathbb{R}^{n}$ be a semialgebraic curve and $x_{0} \in X$. By the standard conic structure theorem, we have the following statement:

There exists a neighbourhood $U_{x_{0}}$ of $x_{0}$ in $\mathbb{R}^{n}$ such that $X \cap U_{x_{0}}=$ $\bigcup_{i=1}^{k} X_{i}$

$X_{i}$ where the sets $X_{i}$ have the following properties:

(1) $X_{i}$ is a semialgebraic subset of $\mathbb{R}^{n}$ and homeomorphic to a semisegment $[1,0)$ by a homeomorphism $h_{i}:[1,0) \rightarrow X_{i}$ such that $h_{i}(0)=x_{0}$;

(2) For every $i \neq j, \quad X_{i} \cap X_{j}=\left\{x_{0}\right\}$;

(3) There exists a number $r_{0}$ such that, for every $i$ and $0 \leq r<$ $r_{0}, \quad \#\left(X_{i} \cap S_{r}\left(x_{0}\right)\right)=1$, for every $i$. (Here $S_{r}\left(x_{0}\right)$ means a sphere centred at $x_{0}$ of radius $r$ ).

Remark 3. The collection $\left\{X_{i}\right\}_{i=1}^{k}$ is a 1-dimensional version of the socalled pancake decomposition of $\left(X, x_{0}\right)$ (see [3], [8], [11]). The elements of this decomposition we call branches (in the similar way to the complex algebraic geometry) or pancakes (in the similar way to the real algebraic geometry).

Let $A$ be a $k$-elements set $A=\left\{a_{1}, \ldots, a_{k}\right\}$. We define a Hölder Semicomplex on $A$ in the following way. Consider a map $x_{i}(r)=X_{i} \cap S_{r}\left(x_{0}\right)$ (for sufficiently small $r$ this function is well defined and semialgebraic). Let $f_{i j}(r)=\left\|x_{i}(r)-x_{j}(r)\right\|$. By the Newton-Puiseux Theorem we obtain, for sufficiently small $r$,

$$
f_{i j}(r)=b_{i j} r^{\alpha_{i j}}+o\left(r^{\alpha_{i j}}\right), \quad \alpha_{i j} \in Q, \quad b_{i j} \in \mathbb{R} .
$$

Put $\alpha\left(a_{i}, a_{j}\right)=\alpha_{i j}$.

Proposition 3.1. $(A, \alpha)$ is a Hölder Semicomplex.

Proof. Since $x_{i}(r)$ and $x_{j}(r)$ belong to $S_{r}\left(x_{0}\right)$, we obtain $f_{i j}(r) \leq 2 r$. Thus, $1 \leq \alpha\left(a_{i}, a_{j}\right)$. Let us prove the isosceles property. Let $X_{1}, X_{2}$ and $X_{3}$ be three different branches of $X$. Let $\alpha\left(a_{1}, a_{2}\right) \leq \alpha\left(a_{2}, a_{3}\right) \leq$ $\alpha\left(a_{1}, a_{3}\right)$. Consider three points $x_{1}(r), x_{2}(r)$ and $x_{3}(r)$. We have:

$$
C_{1} r^{\alpha\left(a_{1}, a_{2}\right)}+o\left(r^{\alpha\left(a_{1}, a_{2}\right)}\right)=\left\|x_{1}(r)-x_{2}(r)\right\| \leq
$$




$$
\begin{aligned}
& \left\|x_{1}(r)-x_{3}(r)\right\|+\left\|x_{2}(r)-x_{3}(r)\right\|= \\
& C_{2} r^{\alpha\left(a_{2}, a_{3}\right)}+o\left(r^{\alpha\left(a_{2}, a_{3}\right)}\right),
\end{aligned}
$$

for some positive constants $C_{1}, C_{2}$. Thus, $\alpha\left(a_{1}, a_{2}\right)=\alpha\left(a_{2}, a_{3}\right)$

The Hölder Semicomplex $(A, \alpha)$ constructed above is called a Hölder Semicomplex associated to $\left(X, x_{0}\right)$. We denote it by $\operatorname{sh}\left(X, x_{0}\right)$.

Let us define another structure associated to $X$ at the point $x_{0}$. Let $X_{i}$ and $X_{j}$ be two branches of $X$. Put

$$
g_{i j}(r)=\operatorname{dist}\left(X_{i}-B_{r}\left(x_{0}\right), X_{j}-B_{r}\left(x_{0}\right)\right)
$$

(here $B_{r}\left(x_{0}\right)$ is the ball centred at $x_{0}$ of radius $r$ ). By the definition of $g_{i j}(r)$ and the quantifier elimination version of the Tarski-Seidenberg Theorem, we obtain that $g_{i j}$ is a semialgebraic function. Thus, by the Newton-Puiseux Theorem,

$$
g_{i j}(r)=c_{i j} r^{\beta_{i j}}+o\left(r^{\beta_{i j}}\right) .
$$

Put $\beta\left(a_{i}, a_{j}\right)=\beta_{i j}$.

Remark 4. Note that $g_{i j}(r)$ is not necessary equal to $f_{i j}(r)$. To see it consider the set $X \subset \mathbb{R}^{2}$ defined as a union of graphs of functions $y=x^{2}$ and $y=x^{3}$ at the point $x_{0}=(0,0)$.

Order Comparison Lemma. For all $i, j$ we have $: \beta_{i j}=\alpha_{i j}$.

Proof. Without loss of generality we can suppose that $x_{0}=0$. Let $v \in \mathbb{R}^{n}$ be a vector such that $\|v\|=1$. Define a cone

$$
C_{\varepsilon}(v)=\left\{u \in \mathbb{R}^{n} ; \angle(u, v)<\varepsilon\right\} .
$$

Suppose that two branches $X_{i}$ and $X_{j}$ have different unit tangent vectors at 0 . Let $v_{i}$ be the unit tangent vector to $X_{i}$ at 0 and $v_{j}$ be the unit tangent vector to $X_{j}$ at 0 . Then there exist $r_{0}>0$ and $\varepsilon>0$ such that, for $r \leq r_{0}$,

$$
X_{i} \cap B_{r}(\mathbf{0}) \subset C_{\varepsilon}\left(v_{i}\right) \cap B_{r}(\mathbf{0}) ;
$$




$$
\begin{aligned}
& X_{j} \cap B_{r}(\mathbf{0}) \subset C_{\varepsilon}\left(v_{j}\right) \cap B_{r}(\mathbf{0}) ; \\
& C_{\varepsilon}\left(v_{i}\right) \cap C_{\varepsilon}\left(v_{j}\right)=\{\mathbf{0}\} .
\end{aligned}
$$

Thus, $g_{i j} \geq \operatorname{dist}\left(C_{\varepsilon}\left(v_{i}\right)-B_{r}(0), C_{\varepsilon}\left(v_{j}\right)-B_{r}(0)\right) \geq c r$, for some $c>0$. It means that $\beta_{i j}=\alpha_{i j}=1$.

Now suppose that $X_{i}$ and $X_{j}$ have the same unit tangent vector $v_{0}$ at 0 . Clearly, $\alpha_{i j} \leq \beta_{i j}$. Suppose that $\alpha_{i j}<\beta_{i j}$. Observe that, for sufficiently small $r$, we can choose the points $y_{i}(r) \in X_{i}$ and $y_{j}(r) \in$ $X_{j}$ such that $g_{i j}(r)=\left\|y_{i}(r)-y_{j}(r)\right\|$ depends semialgebraically and continuously on $r$. Observe that either $y_{i}(r)=x_{i}(r)$, or $y_{j}(r)=x_{j}(r)$, for sufficiently small $r$ (otherwise the function $g_{i j}(r)$ has to be locally constant what contradicts to semialgebraicity). Suppose that $y_{i}(r)=$ $x_{i}(r)$. Consider a triangle with vertices $x_{i}(r), y_{j}(r)$ and $x_{j}(r)$. Since $\beta_{i j}>\alpha_{i j}$, we have:

$$
\left\|x_{i}(r)-y_{j}(r)\right\| \ll\left\|x_{i}(r)-x_{j}(r)\right\|,
$$

for small $r$. Thus, the angle between $x_{i}(r)-x_{j}(r)$ and $y_{j}(r)-x_{j}(r)$ tends to zero as $r$ tends to zero. But, since

$$
\frac{y_{j}(r)-x_{j}(r)}{\left\|y_{j}(r)-x_{j}(r)\right\|} \rightarrow v_{0}, \quad \text { as } \quad r \rightarrow v_{0}
$$

we obtain that $\angle\left(x_{i}(r)-x_{j}(r), v_{0}\right) \rightarrow 0$ as $r \rightarrow 0$.

On the other hand, $X_{i}$ and $X_{j}$ have the same tangent vector $v_{0}$ at $\mathbf{0 .}$ Thus, for every sufficiently small $\varepsilon>0$, there exists $r_{0}>0$ such that, for every $r<r_{0}$, we have $x_{i}(r) \in C_{\varepsilon}\left(v_{0}\right) \cap S_{r}\left(v_{0}\right)$ and $x_{j}(r) \in C_{\varepsilon}\left(v_{0}\right) \cap S_{r}\left(v_{0}\right)$. It means that

$$
\angle\left(x_{i}(r)-x_{j}(r), v_{0}\right) \geq \frac{\pi}{2}-\delta(\varepsilon)
$$

where $\delta(\varepsilon) \rightarrow 0$, as $\varepsilon \rightarrow 0$. This is a contradiction. 


\section{Hölder Semicomplexes as bi-Lipschitz invari- ants}

Theorem 4.1. Germs of closed semialgebraic curves $\left(X_{1}, x_{1}\right)$ and $\left(X_{2}, x_{2}\right)$ are bi-Lipschitz equivalent if and only if the corresponding Hölder Semicomplexes $\left(A_{1}, \alpha_{1}\right)=\operatorname{sh}\left(X_{1}, x_{1}\right)$ and $\left(A_{2}, \alpha_{2}\right)=\operatorname{sh}\left(X_{2}, x_{2}\right)$ are combinatorially equivalent.

Proof. $(\Rightarrow)$ Let $\Phi:\left(X_{1}, x_{1}\right) \rightarrow\left(X_{2}, x_{2}\right)$ be a given bi-Lipschitz map. Let $\left\{X_{i}^{1}\right\}_{i=1}^{k_{1}}$ and $\left\{X_{j}^{2}\right\}_{j=1}^{k_{2}}$ be pancake decompositions of $\left(X_{1}, x_{1}\right)$ and $\left(X_{2}, x_{2}\right)$. Since $\Phi$ is a homeomorphism, we have $k_{1}=k_{2}=k$ and, for each $i, \Phi\left(X_{i}^{1}\right)=X_{i}^{2}$ (we can choose another renumeration if necessary). Let $\left(A_{1}, \alpha_{1}\right)$ and $\left(A_{2}, \alpha_{2}\right)$ be the Hölder Semicomplexes corresponding to $\left(X_{1}, x_{1}\right)$ and $\left(X_{2}, x_{2}\right)$. Let $A_{1}=\left\{a_{i}^{1}\right\}_{i=1}^{k}$, let $A_{2}=\left\{a_{i}^{2}\right\}_{i=1}^{k}$ and put $h\left(a_{i}^{1}\right)=a_{i}^{2}$. Let us prove that $\alpha_{1}\left(a_{i}^{1}, a_{j}^{1}\right)=\alpha_{2}\left(a_{i}^{2}, a_{j}^{2}\right)$, for each $i, j$. Since $\Phi$ is a bi-Lipschitz map, there exists $K>0$ such that, for sufficiently small $r$, we have:

$$
\begin{aligned}
& \Phi\left(X_{i}^{1}-B_{r}\left(x_{1}\right)\right) \subset X_{i}^{2}-B_{K r}\left(x_{2}\right), \\
& \Phi\left(X_{j}^{1}-B_{r}\left(x_{1}\right)\right) \subset X_{j}^{2}-B_{K r}\left(x_{2}\right) .
\end{aligned}
$$
that

Let $y_{i}^{1}(r) \in X_{i}^{1}-B_{r}\left(x_{1}\right)$ and $y_{j}^{1}(r) \in X_{j}^{1}-B_{r}\left(x_{1}\right)$ be points such

$$
\left\|y_{i}^{1}(r)-y_{j}^{1}(r)\right\|=\operatorname{dist}\left(X_{i}^{1}-B_{r}\left(x_{1}\right), X_{j}^{1}-B_{r}\left(x_{1}\right)\right)
$$

Thus, by (1),(2) we obtain:

$$
\left\|\Phi\left(y_{i}^{1}(r)\right)-\Phi\left(y_{j}^{1}(r)\right)\right\| \geq g_{i j}^{2}(K r)
$$

(here $g_{i j}^{1}, g_{i j}^{2}$ mean the function $g_{i j}$ defined for $X_{1}$ and $X_{2}$ correspondingly, see section 3). Since $\Phi$ is a bi-Lipschitz map, there exists a constant $L>0$ such that

$$
g_{i j}^{1}(r)=\left\|y_{i}^{1}(r)-y_{j}^{1}(r)\right\| \geq L \| \Phi\left(y_{i}^{1}(r)\right)-\Phi\left(y_{j}^{1}(r) \| .\right.
$$

Thus,

$$
g_{i j}^{1}(r) \geq L g_{i j}^{2}(K r)
$$


Using the Newton-Puiseux Theorem and the Order Comparison Lemma, we obtain that $\alpha_{1}\left(a_{i}^{1}, a_{j}^{1}\right) \leq \alpha_{2}\left(a_{i}^{2}, a_{j}^{2}\right)$. Using the fact that $\Phi^{-1}$ is also a bi-Lipschitz map we obtain that $\alpha_{1}\left(a_{i}^{1}, a_{j}^{1}\right) \geq \alpha_{2}\left(a_{i}^{2}, a_{j}^{2}\right)$. The first part of the theorem is proved.

$(\Leftarrow)$ Let $\left(A_{1}, \alpha_{1}\right)$ and $\left(A_{2}, \alpha_{2}\right)$ be combinatorially equivalent. We suppose that the isomorphism preserves the enumeration. Let $\left\{X_{i}^{1}\right\}_{i=1}^{k_{1}}$ and $\left\{X_{i}^{2}\right\}_{i=1}^{k_{2}}$ be pancake decompositions of $\left(X_{1}, x_{1}\right)$ and $\left(X_{2}, x_{2}\right)$. Let $r_{0}>0$ be a number such that $x_{i}^{1}(r)$ and $x_{i}^{2}(r)$ are well defined semialgebraic functions, for every $r<r_{0}$ and for every $i$. Consider the semialgebraic function $r: X_{1} \cup X_{2} \rightarrow \mathbb{R}$ defined by

$$
r(x)= \begin{cases}\left\|x-x_{1}\right\|, & \text { for } \quad x \in X_{1} \\ \left\|x-x_{2}\right\|, & \text { for } \quad x \in X_{2} .\end{cases}
$$

Define $\Phi: X_{1} \cap B_{r_{0}}\left(x_{1}\right) \rightarrow X_{2} \cap B_{r_{0}}\left(x_{2}\right)$ by $\Phi(x)=x_{i}^{2}(r(x))$, for $x \in X_{i}^{1}$.

Claim. There exists $\delta>0$ such that $\left.\Phi\right|_{X_{1} \cap B_{\delta}\left(x_{1}\right)}$ is a bi-Lipschitz map.

Proof of the claim. Each $X_{i}^{1}$ and $X_{i}^{2}$ has a tangent vector at $x_{1}$ and $x_{2}$, respectively, hence, for some $\delta_{0}>0$, we have: $\left.\Phi\right|_{X_{i}^{1} \cap B_{\delta_{0}}\left(x_{1}\right)}$ is a bi-Lipschitz map, for all $i$. It is enough to prove that, for each pair $\left(X_{i}^{1}, X_{j}^{1}\right)$, the map $\left.\Phi\right|_{\left(X_{i}^{1} \cup X_{j}^{1}\right) \cap B_{\delta_{1}}\left(x_{1}\right)}$ is bi-Lipschitz, for some $\delta_{1}>0$.

Let $x \in X_{i}^{1}$ and $y \in X_{j}^{1}$ be two points sufficiently close to $x_{1}$. We can suppose that $r(x) \leq r(y)$. Let $z \in X_{j}^{1}$ such that $r(z)=r(x)$. Consider the triangles $(x, y, z)$ and $(\Phi(x), \Phi(y), \Phi(z))$. Since the curves $X_{i}^{1}, X_{j}^{1}, X_{i}^{2}, X_{j}^{2}$ are semialgebraic sets, for sufficiently small $r$, they are close enough to their tangent vectors. Thus, the angle at the vertex $z$ in the triangle $(x, y, z)$ and the angle at the vertex $\Phi(z)$ in the triangle $(\Phi(x), \Phi(y), \Phi(z))$ are bounded away from zero. Using this fact we obtain that there exist $K_{1}, K_{2}>0$ such that

$$
K_{1} \max \left(r(y)-r(x), f_{i j}^{1}(r(x))\right) \leq\|y-x\|
$$

and

$$
\|y-x\| \leq K_{2} \max \left(r(y)-r(x), f_{i j}^{1}(r(x))\right)
$$

(here $f_{i j}^{1}, f_{i j}^{2}$ mean the function $f_{i j}$ defined for $X_{1}$ and $X_{2}$ correspondingly, see section 3). By the same way, we obtain that there exist 
$L_{1}, L_{2}>0$ such that

$$
L_{1} \max \left(r(\Phi(y))-r(\Phi(x)), f_{i j}^{2}(r(\Phi(x)))\right) \leq \| \Phi(y)-\Phi(x) \mid
$$

and

$$
\|\Phi(y)-\Phi(x)\| \leq L_{2} \max \left(r(\Phi(y))-r(\Phi(x)), f_{i j}^{2}(r(\Phi(x))) .\right.
$$

Since $\alpha_{1}\left(a_{i}^{1}, a_{j}^{1}\right)=\alpha_{2}\left(a_{i}^{2}, a_{j}^{2}\right)$, there exist constants $M_{1}, M_{2}>0$ such that

$$
M_{1} f_{i j}^{1}(r) \leq f_{i j}^{2}(r) \leq M_{2} f_{i j}^{1}(r) .
$$

Using the inequalities (3),(4),(5),(7) and the facts that $r(y)=r(\Phi(y))$ and $r(x)=r(\Phi(x))$ we obtain that $\Phi$ is a bi-Lipschitz map. The claim is proved. And, thus, Theorem 4.1 is proved.

\section{The Realization Theorem}

Theorem 5.1. Let $(A, \alpha)$ be a Hölder Semicomplex. Let \#A=k. Then there exists a semialgebraic subset $X \subset \mathbb{R}^{2}$ with $\operatorname{dim} X=1$ satisfying the following conditions:

(1) $(A, \alpha)$ is a Hölder Semicomplex corresponding to $(X, 0)$;

(2) $(X, 0)$ has a pancake decomposition $\left\{X_{i}\right\}_{i=1}^{k}$ such that $X_{i}$ is a graph of an algebraic function $\psi_{i}:[0, \varepsilon] \rightarrow \mathbb{R}$ with $\psi_{i}(0)=0$.

Remark 5. This theorem means that each Hölder Semicomplex can be realized as a plane semialgebraic curve. $X$ is called a realization of $(A, \alpha)$.

Proof of the theorem 5.1. We use induction on $k$. For $k=1$, the statement is obvious. Suppose that the statement is proved, for some $k$. Let $(A, \alpha)$ be a Hölder Semicomplex such that $\# A=k+1$. Let $\alpha_{0}=\max _{i, j} \alpha\left(a_{i}, a_{j}\right)$. We can suppose that $\alpha_{0}=\alpha\left(a_{k}, a_{k+1}\right)$. Put $\tilde{A}=A-\left\{a_{k+1}\right\}$ and $\tilde{\alpha}=\left.\alpha\right|_{\tilde{A} \times \bar{A}}$. Let $\tilde{X}$ be a realization of $(\tilde{A}, \tilde{\alpha})$. Let $\tilde{X}_{i}$ be a pancake corresponding to $a_{\imath}(i=1, \ldots, k)$. By the induction hypothesis, we have that $\tilde{X}_{i}$ is a graph of some algebraic function $\tilde{\psi}_{i}$ : $\left[0, \varepsilon_{0}\right] \rightarrow \mathbb{R}$ such that $\tilde{\psi}_{i}(0)=0$. Put 


$$
\psi_{k+1}(x)=s x^{\alpha_{0}}+\tilde{\psi}_{k}(x) .
$$

Since $\tilde{X}$ contains a finite number of branches, there exist $s$ and $\varepsilon<\varepsilon_{0}$ such that $\psi_{k+1}(x) \neq \tilde{\psi}_{i}(x)$, for every $i=1, \ldots, k$, if $x \neq 0$.

Put $\psi_{i}=\left.\tilde{\psi}_{i}\right|_{[0, \varepsilon]}$ and let $X_{i}$ be the graph of $\psi_{i}$. Let $X=\bigcup_{i=1}^{k+1} X_{i}$. By straightforward calculations we obtain that $(A, \alpha)$ is a Hölder Semicomplex corresponding to $(X, 0)$.

Corollary 5.2. Every semianalytic subset $X \subset \mathbb{R}^{n}$ such that $\operatorname{dim} X=1$ is locally bi-Lipschitz equivalent to some semialgebraic subset $\tilde{X} \subset \mathbb{R}^{2}$.

\section{Hölder Semicomplexes and Hölder Complexes}

Here we are going to compare the bi-Lipschitz invariants of germs of semialgebraic curves defined in this paper with bi-Lipschitz invariants of germs of semialgebraic surfaces (see [1]). A complete intrinsic biLipschitz invariant of two-dimensional semialgebraic sets is called Hölder Complex. Hölder Complex is a pair $(\Gamma, \beta)$ where $\Gamma$ is a finite graph and $\beta$ is a rational valued function defined on the set of edges of $\Gamma$. One can find all the definitions and results related to this subject in [1] and [6].

Let $(\Gamma, \beta)$ be a Hölder Complex. A vertex $a$ of $\Gamma$ is called essential if, for every neighbourhood $U_{a}$ of $a$, we have: $U_{a}$ is not a topological 1manifold (in other words, $a$ is neither artificial nor a loop vertex of $\Gamma$, see $[1])$. We are going to define a Hölder Semicomplex $(\widetilde{A}, \tilde{\alpha})$ corresponding to $(\Gamma, \beta)$ in the following way: $\Gamma$.

(1) The set $V_{\widetilde{A}}$ of vertices of $\widetilde{A}$ be the set of all essential vertices of

(2) Let $a_{1}, a_{2}$ be vertices of $\tilde{A}$. Let $P$ be the set of all finite paths $\gamma=\left\{g_{1}, \ldots, g_{s}\right\}$ (here $g_{1}, \ldots, g_{s} \in E_{\Gamma}$ ) connecting $a_{1}$ and $a_{2}$. We define

$$
\tilde{\alpha}\left(a_{1}, a_{2}\right)=\left\{\begin{array}{l}
1, \text { if } a_{1}, a_{2} \text { belong to different connected components of } \Gamma \\
\max _{\gamma \in P} \min _{g_{k} \in \gamma} \beta\left(g_{k}\right) .
\end{array}\right.
$$




\section{Proposition 6.1. $(\widetilde{A}, \tilde{\alpha})$ is a Hölder Semicomplex.}

Proof. Let us prove the isosceles property. Let $a_{1}, a_{2}, a_{3}$ be three vertices of $\tilde{A}$ such that $\tilde{\alpha}\left(a_{1}, a_{2}\right) \leq \tilde{\alpha}\left(a_{2}, a_{3}\right) \leq \tilde{\alpha}\left(a_{1}, a_{3}\right)$. We can suppose that $a_{1}, a_{2}, a_{3}$ belong to the same connected component of $\Gamma$. (Otherwise the proposition is trivial). Let $\left\{g_{1}, \ldots, g_{k}\right\}$ be a path in $\Gamma$ connecting $a_{1}$ and $a_{2}$. Let $\left\{g_{1}^{\prime}, \ldots, g_{s}^{\prime}\right\}$ be a path connecting $a_{1}$ and $a_{3}$. Clearly, $\left\{g_{k}, \ldots, g_{1}, g_{1}^{\prime}, \ldots, g_{s}^{\prime}\right\}$ is a path connecting $a_{2}$ and $a_{3}$. Thus, $\tilde{\alpha}\left(a_{2}, a_{3}\right) \leq \min \left\{\tilde{\alpha}\left(a_{1}, a_{2}\right), \tilde{\alpha}\left(a_{1}, a_{3}\right)\right\}$. Since $\tilde{\alpha}\left(a_{1}, a_{2}\right) \leq \tilde{\alpha}\left(a_{1}, a_{3}\right)$ we obtain $\tilde{\alpha}\left(a_{2}, a_{3}\right)=\tilde{\alpha}\left(a_{1}, a_{2}\right)$.

Let $Y \subset \mathbb{R}^{n}$ be a two-dimensional semialgebraic set. Let $Y=$ $Y^{0} \cup Y^{1} \cup Y^{2}$ be the canonical topological stratification of $Y$. This stratification can be obtained in the following way. Let $Y^{2}$ be the set of all points $y \in Y$ such that there exists a neighbourhood $U_{y} \subset \mathbb{R}^{n}$ such that $U_{y} \cap Y$ is a topological two-dimensional manifold. Let $Y^{1}$ be the set of points of $Y-Y^{2}$ such that, for each $\tilde{y} \in Y^{1}$, there exists a neighbourhood $U_{\tilde{y}}$ such that $U_{\tilde{y}} \cap\left(Y-Y^{2}\right)$ is a topological one-dimensional manifold. Set $Y^{0}=Y-Y^{2}-Y^{1}$. By [5], $Y^{0}, Y^{1}$ and $Y^{2}$ are semialgebraic sets and, thus, $Y^{0}$ is finite (see also [9]).

Let $y_{0} \in Y^{0}$ and $y_{0} \in C l\left(Y^{1}\right)$ (here $C l\left(Y^{1}\right)$ means a closure of $\left.Y^{1}\right)$. Then the germ of $C l\left(Y^{1}\right)$ at $y_{0}$ is a germ of one-dimensional semialgebraic set. Let $(A, \alpha)$ be a Hölder Semicomplex associated to $\left(C l\left(Y^{1}\right), y_{0}\right)$ (i.e. $\left.(A, \alpha)=\operatorname{sh}\left(C l\left(Y^{1}\right), y_{0}\right)\right)$. Let $(\Gamma, \beta)$ be a canonical Hölder Complex of $Y$ at $y_{0}$ (see[1]). Let $(\widetilde{A}, \tilde{\alpha})$ be the Hölder Semicomplex corresponding to $(\Gamma, \beta)$ defined above.

\section{Theorem 6.2.}

(1) There exists a morphism $\mathrm{m}:(\tilde{A}, \tilde{\alpha}) \rightarrow(A, \alpha)$.

(2) If $Y$ is locally normally embedded at $y_{0}$ (see [3]) then $(\tilde{A}, \tilde{\alpha})$ and $(A, \alpha)$ are isomorphic.

Proof. (2) Let $Y_{i}^{1}$ and $Y_{j}^{1}$ be two branches (pancakes) of $C l\left(Y^{1}\right)$. Let $a_{i}$ and $a_{j}$ be the corresponding vertices in $(\Gamma, \beta)$. Suppose that $a_{i}$ and $a_{j}$ belong to the same connected component of $\Gamma$. Let $\gamma=$ $\left\{g_{1}, \ldots, g_{s}\right\}$ be a "maximal" path in $\Gamma$ connecting $a_{i}$ and $a_{j}$ (it means that $\left.\tilde{\alpha}\left(a_{i}, a_{j}\right)=\min _{g_{k} \in \gamma} \beta\left(g_{k}\right)\right)$. By results of [1], the union of the curvilinear triangles corresponding to the edges $g_{1}, \ldots, g_{s}$ is bi-Lipschitz equivalent 
to the standard $\tilde{\alpha}\left(a_{i}, a_{j}\right)$-Hölder triangle. Let $a_{i}(r) \in Y_{i}^{1}$ and $a_{j}(r) \in Y_{j}^{1}$ be points such that

$$
d_{\text {ind }}\left(a_{i}(r), y_{0}\right)=d_{\text {ind }}\left(a_{j}(r), y_{0}\right)=r .
$$

Using results of [1] or [10] we obtain that there exist two constants $K_{1}$ and $K_{2}$ such that

$$
K_{1} d_{l}\left(a_{i}(r), y_{0}\right) \leq r^{\tilde{\alpha}\left(a_{i}, a_{j}\right)} \leq K_{2} d_{l}\left(a_{i}(r), y_{0}\right)
$$

(here $d_{l}$ is the length metric). Since $Y$ is locally normally embedded at $y_{0}$ we obtain (using results of sections 2-4) that $\alpha\left(a_{i}, a_{j}\right)=\tilde{\alpha}\left(a_{i}, a_{j}\right)$. If $a_{i}$ and $a_{j}$ belong to different connected components of $\Gamma$ then $\alpha\left(a_{i}, a_{j}\right)=1$ (because $Y$ is locally normally embedded).

(1) Let $Y$ be not locally normally embedded at $y_{0}$. Let $\tilde{Y}$ be the "normalization" obtained in [3]. Let $\widetilde{Y}^{0} \cup \widetilde{Y}^{1} \cup \widetilde{Y}^{2}$ be a topological canonical stratification of $\tilde{Y}$. Let $\Phi: \tilde{Y} \rightarrow Y$ be a semialgebraic map satisfying the following conditions:

(a) $\Phi$ is a bi-Lipschitz map with respect to the length metric. (b) $\Phi$ is a Lipschitz map with respect to the induced metric. Existence of this map is shown in [3]. Clearly, $\Phi\left(\widetilde{Y}^{1} \cup \widetilde{Y}^{0}\right)=Y^{1} \cup Y^{0}$. Let $\widetilde{Y}_{i}^{1}$ and $\widetilde{Y}_{j}^{1}$ be two branches of $\tilde{Y}^{1}$ and let $\tilde{y}_{0}=\Phi^{-1}\left(y_{0}\right)$. Let $\tilde{a}_{i}(r) \in \tilde{Y}_{i}^{1}$ and $\tilde{a}_{j}(r) \in \tilde{Y}_{j}^{1}$ be two points such that

$$
d_{\text {ind }}\left(\tilde{a}_{i}(r), \tilde{y}_{0}\right)=d_{\text {ind }}\left(\tilde{a}_{j}(r), \tilde{y}_{0}\right)=r .
$$

By [3], we have

$$
d_{\text {ind }}\left(\tilde{a}_{i}(r), \tilde{a}_{j}(r)\right) \geq d_{\text {ind }}\left(a_{i}(r), a_{j}(r)\right),
$$

for sufficiently small $r$. Since $\tilde{Y}$ is normally embedded, we obtain that $(\widetilde{A}, \tilde{\alpha})$ is a Hölder Semicomplex associated to $\left(C l\left(\widetilde{Y}^{1}\right), \tilde{y}_{0}\right)$. Since

$$
d_{i n d}\left(\tilde{a}_{i}(r), \tilde{a}_{j}(r)\right)=r^{\tilde{\alpha}\left(a_{i}, a_{j}\right)}+o\left(r^{\bar{\alpha}\left(a_{i}, a_{j}\right)}\right)
$$

and

$$
d_{i n d}\left(a_{i}(r), a_{j}(r)\right)=r^{\alpha\left(a_{i}, a_{j}\right)}+o\left(r^{\alpha\left(a_{i}, a_{j}\right)}\right),
$$

we have that

$$
\tilde{\alpha}\left(a_{i}, a_{j}\right) \leq \alpha\left(a_{i}, a_{j}\right) .
$$


Set $\mathfrak{m}\left(a_{i}\right)=a_{i}$, for all $i$. By (8), $\mathfrak{m}$ is a morphism of Hölder Semicomplexes.

Remark 6. If $Y$ is not normally embedded $(A, \alpha)$ and $(\tilde{A}, \tilde{\alpha})$ are not necessary isomorphic. To see it consider the following example. Let $(\Gamma, \beta)$ be a Hölder Complex such that $\Gamma$ has more than one essential vertices. Let $a_{1}$ and $a_{2}$ be two essential vertices. Let $\Gamma^{\prime}$ be a new graph obtained from $\Gamma$ by adding a new edge $h$ connecting $a_{1}$ and $a_{2}$. Set $\beta^{\prime}(h)>\max _{g \in E_{\Gamma}} \beta(g)$. Then $\left(\Gamma^{\prime}, \beta^{\prime}\right)$ is a Hölder Complex. Using the algorithm from [6] we construct a set $Y$ and a point $y_{0}$ such that $\left(Y, y_{0}\right)$ is a Geometric Hölder Complex corresponding to $(\Gamma, \beta)$. We can suppose that $Y$ is normally embedded (otherwise we can obtain it using [3]). Consider now a semialgebraic set $Y^{\prime}$ obtained from $Y$ by cutting the triangle corresponding to the edge $h$. For this set the Hölder Semicomplexes considered above are not isomorphic.

Proposition 6.3. Let $\left(Y_{1}, y_{1}\right)$ and $\left(Y_{2}, y_{2}\right)$ be two germs of 2-dimensional semialgebraic sets such that $\left(Y_{1}, y_{1}\right)$ and $\left(Y_{2}, y_{2}\right)$ are bi-Lipschitz equivalent with respect to the induced metric. Then the corresponding Hölder Semicomplexes $\left(A_{1}, \alpha_{1}\right)$ and $\left(A_{2}, \alpha_{2}\right)$ are isomorphic.

The proof is straightforward.

\section{References}

[1] Birbrair L. Local bi-Lipschitz classification of 2-dimensional semialgebraic sets. - Houston Journal of Math., N3, vol.25, pp.453-472, 1999.

[2] Bröker L., Kuppe M., Scheufler W. Inner metric properties of 2dimensional semi-algebraic sets. Revista Matematica Complutense.vol.10, pp. 51-78, 1997.

[3] Birbrair L., Mostowski T. Normal embedding of semialgebraic sets. Michigan Math. Journal, vol. 47, pp. 125-132, 2000.

[4] Bochnak J., Risler J-J. Sur les exposants de Lojasiewicz. Comment Math. Helvetici 50, 493-507, 1975.

[5] Benedetti R., Risler J-J. Real algebraic and semialgebraic sets. Hermann, 1990.

[6] Birbrair L., Sobolevsky M. Realization of Hölder Complexes. - Annales de la Faculte des Sciences de Toulouse, N1, vol.VIII, pp.35-44, 1998. 
[7] Gibson C.G., Wirthmüller K., du Plessis A.A., Looijenga E.J.N. Topological stability of smooth mappings. Lecture Notes in Mathematics, vol. 552, Springer, 1976.

[8] Kurdyka K. On a subanalytic stratification satisfying Whitney property with exponent 1. Lecture Notes of Mathematics, 1524, pp. 316-322, 1992.

[9] King H. Topological invariance of intersection homology without sheaves. - Topology and its applications, N20, pp.149-160, 1985.

[10] Kurdyka K., Orro P. Distance geodesique sur un sous-analytique. Revista Matematica Complutence. vol 10. pp.173-182, 1997.

[11] Parusinski A. Lipschitz properties of semianalytic sets. Ann. Inst. Fourier (Grenoble) 38 no. 4, pp. 189-213, 1998.

Lev Birbrair

Departamento de Matemática

Universidade Federal do Ceará

Campus do Pici Bloco 914

CEP 60455-760- Fortaleza, Ce-Brasil

E-mail: lev@mat.ufc.br

Alexandre C.G. Fernandes

Instituto de Matematica

USP - Sao Carlos

Caixa Postal 668, 13560-000

Sao Carlos, SP-Brasil

E-mail: alex@icmc.sc.usp.br

Recibido: 24 de Mayo de 1999

Revisado: 6 de Marzo de 2000 